\title{
QUANTUM COMPUTER ELEMENTS BASED ON COUPLED QUANTUM WAVEGUIDES
}

\author{
M. I. Gavrilov, L. V. Gortinskaya, A. A. Pestov, I. Yu. Popov ${ }^{1}$, E. S. Tesovskaya \\ Department of Higher Mathematics, St. Petersburg State University of Information Technologies, \\ Mechanics and Optics, St. Petersburg, Russia
}

Possible applications of two weakly coupled quantum waveguides for quantum computation are considered. The approach is based on the resonance phenomena in the system. Two different qubits interpretations are described. Some single-qubit and two-qubits operations are realized in the framework of these interpretations.

Предложены возможные применения двух слабосвязанных квантовых волноводов для квантовых вычислений. Используется метод, основанный на резонансных эффектах в системе. Описаны две различные интерпретации квантового бита. Реализованы некоторые однокубитовые и двухкубитовые операции в рамках данных интерпретаций.

\section{INTRODUCTION}

Creation of «hardware» for quantum computer is an interesting problem of nanoscience. There are several ways for realization of quantum computing. It can be based on using of ion traps, nuclear magnetic resonance, superconducting devices, quantum dots, photonic crystals, etc. (see, e.g., $[1,2]$ ). It should be mentioned that in spite of the fact that creation of new quantum algorithms (quantum computer «software») is a mathematical question it is closely related to the physical problems - description of electron (or photon) behaviour in the corresponding quantum systems. Each quantum algorithm (e.g., Shor's algorithm of factorization, Grover's algorithm, etc.) can be considered as a system of basic quantum gates. That is why it is necessary to show how to construct the corresponding gate with using of the chosen quantum system. Moreover, the change of the physical background for quantum computation leads to the change of the answer for the question: "What is a qubit for the system?»

The present paper is devoted to the description of resonant phenomena in coupled quantum waveguides and layers and its applications. Resonant character of the electron transport in weakly coupled waveguides allows one to use the system as a quantum gate. Namely, it is possible to obtain spin-polarized electron beam (preparation of the initial state for quantum computing) and to realize one-qubit and two-qubits operations (Hadamard operator, CNOT, SWAP). The corresponding systems are described in the paper. We don't discuss here the problem of decoherence, it will be a subject of the following publications.

\footnotetext{
${ }^{1}$ E-mail: popov@mail.ifmo.ru
} 


\section{RESONANCES IN COUPLED WAVEGUIDES}

When analyzing the behaviour of ballistic electron in weakly coupled nanowires one reduces the problem to the spectral problem for the Helmholtz operator (the Schrödinger operator for free particle) in the complicated domain with the Dirichlet boundary condition. Some results were obtained earlier. Namely, variational estimates for the bound state (not for resonances) close to the threshold are in $[3,4]$, for the combined Dirichlet-Neumann case are in [8]. Asymptotics of the bound states for the Dirichlet case is in [5,6]. As for resonances (quasi-bound states), the corresponding asymptotics for the Dirichlet case was constructed in [7,8]. Some estimates for the resonances (Dirichlet case) was get in [9]. Natural question concerning the analogous problem for other boundary conditions was open.

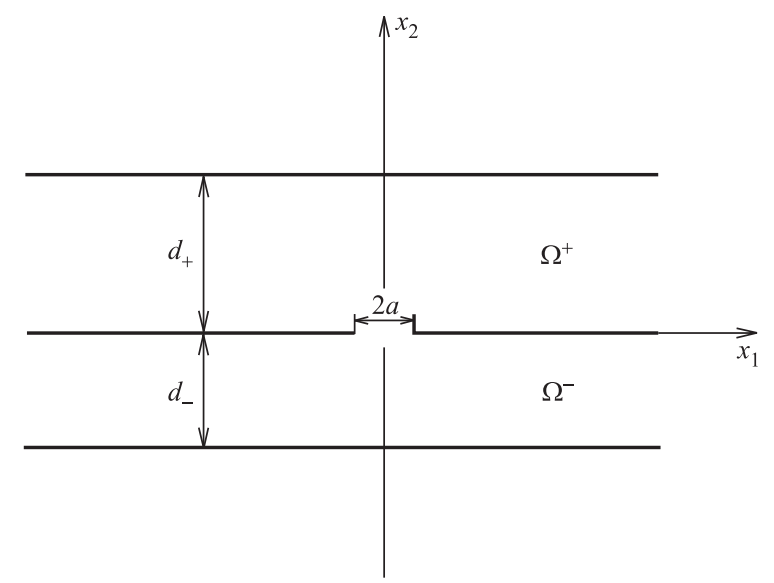

Fig. 1. Geometric configuration of the system

We consider 2D waveguides (see Fig. 1) coupled through small window (or a number of windows). The ballistic electron wave function satisfies the Helmholtz equation $\Delta \psi+k^{2} \psi=$ 0 . We should find quasi-bound state $k_{a}^{2}$ and the corresponding function $\psi$ (it is not an eigenvalue because function $\psi$ doesn't belong to $L_{2}$ space). In the case of the Dirichlet boundary condition, the asymptotics of $k_{a}^{2}$ close to the threshold $\frac{\pi^{2}}{d_{-}^{2}}$ is as follows [9]:

$$
\sqrt{\frac{\pi^{2}}{d_{-}^{2}}-k_{a}^{2}}=k_{20} a^{2}+k_{40} a^{4}+k_{41} a^{4} \log \frac{a}{d_{-}}+o\left(a^{4}\right) .
$$

Expressions for the coefficients see in [9].

In the case of the Neumann boundary condition at the separating line and the Dirichlet condition on the other boundaries, the asymptotics (in small $a$ ) of quasi eigenvalue (resonance) $k_{a}^{2}$ close to the threshold $\frac{9 \pi^{2}}{4 d_{-}^{2}}$ of the corresponding Laplacian has the form [12]:

$$
\sqrt{\frac{9 \pi^{2}}{4 d_{-}^{2}}-k_{a}^{2}}=\tau_{1} \ln ^{-1}\left(a / d_{-}\right)+\tau_{2} \ln ^{-2}\left(a / d_{-}\right)+o\left(\ln ^{-2}\left(a / d_{-}\right)\right) .
$$

Expressions for the coefficients see in [12]. 
Comparing the results (1), (2), one can observe that the asymptotics for the Dirichlet and for the Neumann cases has different orders in respect to $a$. Namely, the influence of the window is greater for the Neumann case. It is natural because unperturbed solution (without a window) has maximum of the absolute value at the boundary in the Neumann case and a node (zero) in the Dirichlet case.

The fact that the resonances have different positions in the Dirichlet and the Neumann cases can be used for construction of nanodevices. It is possible in sandwich structure of magnetic and non-magnetic layers that the wave function of electron with one spin orientation satisfies the Dirichlet boundary condition and, at the same time, the wave function for an electron with another spin orientation satisfies the Neumann condition. It means that for appropriate choice of the parameters one has the resonance for the electron with one spin orientation (i.e. the transmission coefficient is essentially less than 1 and the reflection coefficient is not zero) and the absence of the resonance for the electron with another spin orientation (i.e. the reflection coefficient is zero). Hence, we have a beam of spin polarized reflected electrons. As for the transmitted electron beam, it will be partially spin polarized. If we want to obtain fully transmitted spin polarized electron beam, it is possible to use a system with great number of coupling windows each of them being sufficiently far from others (to avoid interference effects), then the polarization effect accumulates.

\section{QUANTUM DEVICES}

2.1. «Waveguide» Interpretation. Let us assume qubit as a state of electron in two adjacent coupled 2D waveguides. Namely, if the electron is localized in one of these strips (zero-layer) we consider this state as the state $|0\rangle$ and if in the other one (first-layer) as the state $|1\rangle$. Due to the waveguide coupling there are also coherent superpositions of the states (when the wave function of the electron is distributed between the layers). At the same time, it is important that the separating barrier is large enough to maintain the coherence of the qubits during computation.

To prepare the initial state $\left|0_{1}, 0_{2}, 0_{3}, \ldots, 0_{L}\right\rangle$ it is sufficient to place electrons in each zero-layer of qubits. At present time, this preparation can be realized using single electron pump. Such electron pumps are placed near zero-layer of every qubit. At the end of calculations, it is necessary to find the resulting state (by measuring). This operation can be accomplished with single electron transistor attached to the layer. The main disadvantage of this circuit is a big response time of single electron transistor.

Single-Qubit Operations. Realization of single-qubit operation is related to a behaviour of electron in two waveguides coupled thought a window. The states of outgoing and incoming electrons are related by the rotation matrix

$$
R_{x}(\theta)=\left(\begin{array}{cc}
\cos \theta / 2 & i \sin \theta / 2 \\
i \sin \theta / 2 & \cos \theta / 2
\end{array}\right),
$$

where $\theta$ is related to the width of the window and a phase shift. In rough approximation, it is obtained by the expansion of the wave function into the sum of symmetric and antisymmetric functions. Waveguide theory (see, e.g., [13]) allows one to obtain more detailed results.

The other single-qubit operation is the shift-phase of two qubit components (transformation matrix $R_{z}(\theta)$ ). To realize this operation it is necessary to insert a potential barrier (the hight 
of which is less than the electron energy) or well in some layer. Then the phase shift of the wave function is

$$
\varphi_{\text {step }}=n \pi\left(1-\frac{1}{\sqrt{1-V / E}}\right) .
$$

Note that both the window and the potential barrier lead to some wave reflection that gives us some error. Any single-qubit operation can be produced by the combination of operations described above:

$$
U=e^{i \delta} R_{z}(\alpha) R_{x}(\theta) R_{z}(\beta) .
$$

For example, the Hadamard's gate is described as

$$
H=-i R_{z}\left(\frac{\pi}{2}\right) R_{x}\left(\frac{\pi}{2}\right) R_{z}\left(\frac{\pi}{2}\right) .
$$

Two-Qubits Operations. To perform two-qubits operation it is necessary to synchronize the movement of the electrons in different channels. Let us consider the coupling window located between two layers corresponding to different qubits. To describe this operation one should consider two-particles problem. The first approximation gives us the problem of particle in the field created by the second electron. It should be stressed that the resonance peaks are very sharp and the position of the resonance for this case differs from that for the single-electron case. It is possible to realize the situation when for the chosen energy of the electron one has the resonance in the two-particle case and the absence of the resonance in the single-electron case. Let the first-layers of two qubits be coupled through the window. For this case, the transformation matrix is

$$
\mathbf{e}^{i \gamma}=\left(\begin{array}{cccc}
1 & 0 & 0 & 0 \\
0 & 1 & 0 & 0 \\
0 & 0 & 1 & 0 \\
0 & 0 & 0 & e^{i \gamma}
\end{array}\right)
$$

where the value of the parameter is determined by the window width.

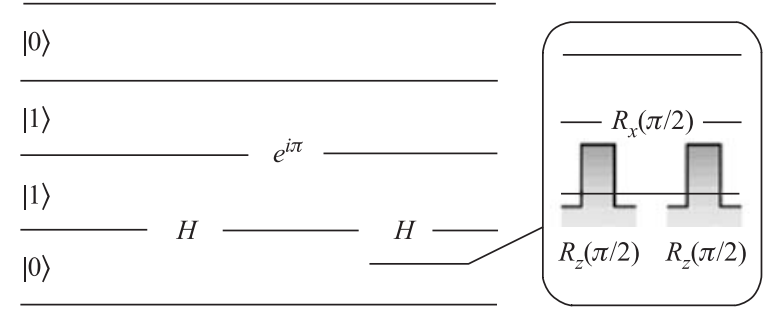

Fig. 2. CNOT in «waveguide» interpretation

Using this operation and the Hadamard's gate we can easily construct CNOT gate (see Fig. 2):

$$
\mathrm{CNOT}=(1 \otimes H) \mathbf{e}^{i \pi}(1 \otimes H) .
$$

Due to the resonance existence strong coupling is observed for small window. Moreover, the resonance range is very narrow and weak variation of the window width can destroy or 
create the resonance. The window size can be slightly varied by bias voltage with using of MOS structures.

2.2. «Spin» Interpretation. Let us assume qubit as electron spin direction: up-spin electron corresponds to the state $|0\rangle$, and the down-spin electron to the state $|1\rangle$. It is necessary to initialize quantum register before calculations. Initializing can be made by passing electron through a spin-filter. There are many types of these filters, such as: one using special magnetic materials [14], filters based on Rashba's effect [15], quantum dots of special structure [16].

Sandwich structure of weakly coupled magnetic/nonmagnetic conducting layers can be used as a spin-filter. Let the magnetic layers be narrow. The nonmagnetic layers can be considered as waveguides. The boundary conditions are determined by the number of free levels in magnetic layers with given spin orientation of the electron. If there are no free levels for the spin orientation, then one has the Dirichlet condition at the boundary. In other cases, we have another boundary conditions, for example, the Neumann one. Due to the difference in resonance position for different boundary conditions and the narrowness of the resonant peak there exist electron energies corresponding to the resonance for one spin orientation and the absence of the resonance for another one. Thus, the spin polarized beam of reflected electrons appears, and the transmitted beam is partly polarized. The system of some coupling windows (which are placed at a distance to prevent an appearance of complex interference effects) gives us a strongly polarized beam. The same structure can be used for final measuring of resulting system state. Single-qubit operations can be accomplished as follows. We can use the applied electromagnetic field in some domains or ferromagnetic inclusions inserted in some parts of the semiconductor boundary.

Let us consider the first method [17]. Even with the absence of external magnetic fields, spin rotation of a mobile electron can still be achieved with the so-called spin-orbit interaction. An electron moving with velocity $v$ in a region with a static electric field $E$ is forced by the effective magnetic field $B \sim v \times E$ which couples with its spin. The spin-orbit Hamiltonian due to this coupling is $H \sim \boldsymbol{\sigma}(k \times E)$, where $k$ is the electron wave-vector and $\boldsymbol{\sigma}=\left(\sigma_{x}, \sigma_{y}, \sigma_{z}\right)$ is the vector of Pauli matrices. The Hamiltonian $H$ contains the necessary ingredients for implementing spin rotations around two independent axes and, therefore, an arbitrary single-qubit operation.

Two-qubit operations are accomplished during the interaction of two synchronously passing electrons in the coupling region. The Hamiltonian of the spin exchange interaction is $H_{\mathrm{ex}}=J(t) S_{1} S_{2}$, where $J(t)$ is defined by the overlapping of the electrons wave functions. The simplest model of spin-spin interaction can be obtained in the framework of zero-range potential method [18]. If interaction time $T$ satisfies the condition

$$
\int_{0}^{T} \frac{J(t)}{\hbar} d t=\pi,
$$

then SWAP operation will be executed with the qubits. If the time of the interaction is such that

$$
\int_{0}^{T} \frac{J(t)}{\hbar} d t=\frac{\pi}{2},
$$


then the resulting operation is

$$
\begin{aligned}
& \sqrt{\mathrm{SWAP}}=(2 i)^{-1 / 2}\left(\begin{array}{cccc}
(2 i)^{1 / 2} & 0 & 0 & 0 \\
0 & i & 1 & 0 \\
0 & 1 & i & 0 \\
0 & 0 & 0 & (2 i)^{1 / 2}
\end{array}\right) .
\end{aligned}
$$

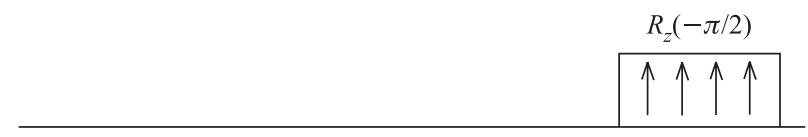

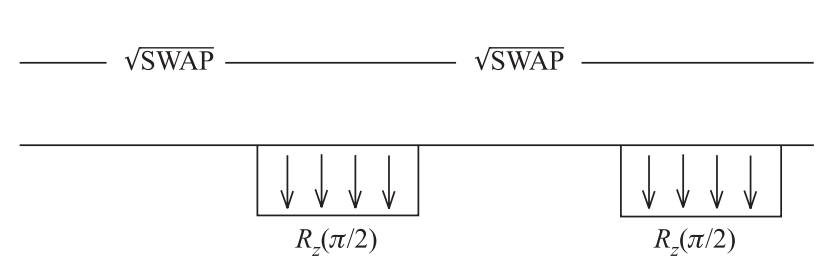

Fig. 3. CNOT in «spin» interpretation

The single-qubit operation $R_{z}$ with $\sqrt{\mathrm{SWAP}}$ allows one to implement gate CNOT by the following composition of operations (see Fig. 3):

$$
\mathrm{CNOT}=\left(R_{z}^{A}(\pi / 2) \otimes R_{z}^{B}(-\pi / 2)\right) \sqrt{\operatorname{SWAP}}\left(R_{z}^{A}(\pi / 2) \otimes 1^{B}\right) \sqrt{\operatorname{SWAP}} .
$$

Here the indices $A$ and $B$ correspond to the first and the second qubits, respectively. Thus, we come to complete operations system.

There are some difficulties with the realization of the interaction of any qubit pair. To perform the operation with any of two qubits it is necessary to localize the corresponding electrons in the adjacent waveguides. Using of some SWAP operations allows us to shift the electrons into the neighbor waveguides.

Implementation of two-qubit operations based on the spin exchange interaction gives us another possibility to realize the single-qubit operations. In this case, ferromagnetic inclusion is inserted in some part of the semiconductor boundary. The length of this section predetermines the rotation angle in the single qubit state space.

When we use the «waveguide» interpretation we deal with spinless particles and it is not necessary to prepare spin-polarized beam of electron, but there are difficulties with the localization of the electrons in particular waveguides. For using of the «spin» interpretation one should prepare the initial spin-polarized electron beam that can be created by the spin-filter described above. As for the comparison of two suggested qubit interpretations, the «spin» one seems to be preferable for realization.

Acknowledgements. The work is supported by Russian Foundation for Basic Researches (grant 05-03-32576) and Program of Integration of Science and High Education (State contract No. П0045/2307). 


\section{REFERENCES}

1. Valiev K. A., Kokin A. A. Quantum Computers: Hopes and Reality. R\&C Dynamics. Izhevsk, 2001.

2. Barenco A. et al. // Phys. Rev. A. 1995. V.52. P. 3457.

3. Exner P., Vugalter S. // Ann. Inst. Henri Poincare. 1996. V.65. P. 109.

4. Exner P., Vugalter S. // J. Phys. A: Math. Gen. 1997. V. 30. P. 7863.

5. Popov I. Yu. // Rep. Math. Phys. 1999. V.43. P. 427.

6. Popov I. Yu. // Theor. Math. Phys. 2002. V.131. P. 407.

7. Popov I. Yu. // J. Math. Phys. 2002. V. 43. P. 215.

8. Dittrich J., Kriz J. // Ibid. P. 3892.

9. Frolov S. V., Popov I. Yu. // J. Math. Phys. 2000. V.41. P. 4391.

10. Frolov S. V., Popov I. Yu. // J. Phys. A: Math. Gen. 2003. V.36. P. 1655.

11. Kunze Ch. // Phys. Rev. B. 1993. V. 48. P. 14338.

12. Gortinskaya L.V., Popov I. Yu., Tesovskaya E.S. // Proc. of Intern. Seminar «Day on Diffraction'2003» St. Petersburg, 2003. P. 52.

13. Mittra R., Lee S. W. Analytical Methods in the Theory of Guided Waves. McMillan and Company, 1971.

14. Taddei F., Sanvito S., Lambetr C.J. cond-mat/0012352. 2000. P. 4.

15. Ramaglia V. et al. cond-mat/0203569. 2002. P. 16.

16. Recher P., Sukhorukov E. V., Loss D. // Phys. Rev. Lett. 2000. V. 85. P. 1962.

17. Popescu A. E., Ionicioiu R. // Phys. Rev. B. 2004. V.69. P.245422.

18. Avdonin S. A. et al. // J. Phys. A: Math. Gen. 2005. V.38. P. 4825. 